\title{
Características Fisiológicas de Cultivares de Mandioca após AplicaÇão do MEsotrione ${ }^{1}$
}

\author{
Physiological Characteristics of Cassava Cultivars after Mesotrione Application
}

\author{
SILVEIRA, H.M. ${ }^{2}$, FERREIRA, E.A. ${ }^{3}$, SILVA, D.V. ${ }^{4}$, NETO, M.D.C. ${ }^{5}$, CARVALHO, F.P. ${ }^{6}$, \\ SANTOS, J.B. ${ }^{7}$ e SILVA, A.A. ${ }^{8}$
}

\begin{abstract}
RESUMO - Neste trabalho, avaliou-se a taxa fotossintética e a eficiência do uso da água em cultivares de mandioca (Coimbra, Platina, IAC-12, Coqueiro e Cacau-UFV), pulverizados com cinco doses do mesotrione $\left(0,72,108,144\right.$ e $\left.216 \mathrm{~g} \mathrm{ha}^{-1}\right)$. Aos 45 dias após aplicação do herbicida, foram avaliados $\mathrm{O} \mathrm{CO}_{2}$ consumido, o carbono interno, a taxa fotossintética, a condutância estomática de vapores de água, a taxa de transpiração, a temperatura da folha e a eficiência do uso da água. O cultivar Platina mostrou-se menos afetado pelo mesotrione. Apenas neste cultivar as doses aplicadas de 144 e $216 \mathrm{~g} \mathrm{ha}^{-1}$ não alteraram a taxa fotossintética das plantas. A temperatura da folha dos cultivares de mandioca também não foi alterada pelo herbicida; entretanto, observou-se redução na eficiência de uso da água para o cultivar Coqueiro.
\end{abstract}

Palavras-chave: eficiência do uso da água, Manihot esculenta, taxa fotossintética.

\begin{abstract}
Efficiency of the photosynthetic rate and water use was evaluated in cassava cultivars (Coimbra, Platina, IAC-12, Coqueiro, and Cacau-UFV), sprayed with five mesotrione rates (0, 72, 108,144 , and $\left.216 \mathrm{~g} \mathrm{ha}^{-1}\right) . \mathrm{CO}_{2}$ consumed, internal carbon, photosynthetic rate, water vapor stomatal conductance, transpiration rate, leaftemperature, and water use efficiency were assessed 45 days after application of the herbicide. Platina cultivar was found to be less affected by mesotrione. Only in this cultivar, the applied rates of 144 and $216 \mathrm{~g} \mathrm{ha}^{-1}$ did not alter the photosynthetic rate of the plants. The leaf temperature of the cassava cultivars was not affected by the herbicide, either; however, reduced water use efficiency was observed in the Coqueiro cultivar.
\end{abstract}

Keywords: water use efficiency, Manihot esculenta, photosynthetic rate

\section{INTRODUÇÃO}

Apesar de o Brasil ser o terceiro maior produtor de mandioca (Manihot esculenta) no mundo, são poucas as opções de defensivos agrícolas para a cultura. Isso tem dificultado reduções nos custos de produção e causado perdas em plantios comerciais (ABAM, 2011).
O manejo de plantas daninhas é tido como um dos principais componentes de produção da mandioca, principalmente devido às reduções na produção de raízes, que pode chegar a mais de 90\% (Johanns \& Contiero, 2006). O manejo químico tem sido indicado como a alternativa para reduzir o custo final do cultivo, em razão do alto custo e baixo rendimento da

Recebido para publicação em 1.8.2012 e aprovado em 12.10.2012.

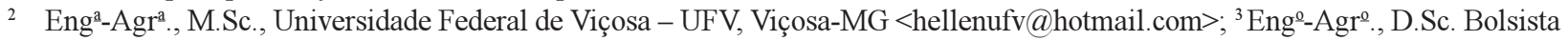
PNPD, Universidade Federal dos Vales do Jequitinhonha e Mucuri - UFVJM, Diamantina-MG, ${ }^{4}$ Eng $^{\mathrm{o}}$-Agr ${ }^{\mathrm{o}}$., Doutorando, Programa de Pós-Graduação em Fitotecnia, Departamento de Fitotecnia, Universidade Federal de Viçosa-DFT/UFV; ${ }^{5}$ Eng $^{-0}$-Agr ${ }^{0}$., Doutorando, Programa de Pós-Graduação em Fitotecnia - DFT/UFV; ${ }^{6}$ Engọ-Agr $^{\circ}$, Doutorando, Programa de Pós-Graduação em Fitotecnia DFT/UFV; ${ }^{7}$ Engo-Agr ${ }^{\circ}$., D.Sc., Professor Adjunto, Departamento de Agronomia - UFVJM; ${ }^{8}$ Engo-Agro ${ }^{-}$, D. Sc., Professor Associado, DFT/UFV.

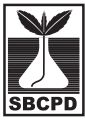

Planta Daninha, Viçosa-MG, v. 31, n. 2, p. 403-409, 2013 
capina com enxada (Biffe et al., 2010). No entanto, a indisponibilidade de produtos registrados para casos específicos tem sido o entrave ao crescimento do setor.

Entre os herbicidas com potencial de uso na cultura da mandioca, destaca-se o mesotrione. Esse herbicida inibe a biossintese de carotenoides, interferindo na atividade da enzima HPPD (4 - hidroxifenilpiruvato - dioxigenase) nos cloroplastos, causando o branqueamento com posterior necrose e morte dos tecidos vegetais em cerca de uma a duas semanas (Lee, 1997; Witchert et al., 1999). Quando aplicado em pós-emergência inicial, tem promovido excelente controle de várias espécies de plantas daninhas, como: Digitaria horizontalis, Eleusine indica, Brachiaria plantaginea, Acanthospermum hispidum, Portulaca oleracea e Galinsoga parviflora (Foloni, 2002). Registrado para a cultura do milho, a tolerância dessa espécie ao herbicida tem sido atribuída à capacidade de metabolizar rapidamente, produzindo metabólitos sem atividade tóxica (Syngenta, 2012). A absorção do produto ocorre tanto nas raízes quanto nas folhas e ramos (Brasil, 2012).

Acredita-se que uma das características fisiológicas das plantas suscetiveis influenciada pelo uso de herbicidas seja a taxa fotossintética. Silveira et al. (2012) observaram que, em geral, plantas de mandioca tratadas com mesotrione mantiveram o aumento do número de folhas durante o período de avaliação apesar da intoxicação visível. Essa é uma característica desejada, pois a manutenção da folhagem das plantas de mandioca aumenta o período de atividade do aparato fotossintético, proporcionando incrementos no rendimento da produção da cultura.

Outra característica fisiológica possivel de ser afetada pelo uso de herbicidas é a eficiência do uso da água pela planta. Dessa forma, plantas mais eficientes no uso da água produzem mais matéria seca por grama de água transpirada (Baptista et al., 2001). Para estudos de competição com plantas daninhas, Aspiazú et al. (2010) evidenciaram que, na ausência de outra planta, a mandioca foi mais eficiente quanto ao uso da água, sobretudo quando competindo com Bidens pilosa. Esta espécie mostrou ter maior capacidade de afetar o crescimento da cultura (Procópio et al., 2004).
Na busca de novos herbicidas seletivos à cultura da mandioca, foram avaliadas neste trabalho características associadas à eficiência fotossintética e ao uso da água em cultivares dessa cultura, submetidos a doses crescentes do mesotrione.

\section{MATERIAL E MÉTODOS}

O experimento foi realizado em ambiente protegido, sendo utilizado como substrato amostra de Latossolo Vermelho-Amarelo distrófico típico (LVAd), textura argilosa (56\% de argila, $6 \%$ de silte e $38 \%$ de areia). A análise química do solo apresentou $\mathrm{pH}$ (água) de 5,4; teor de matéria orgânica de 1,8 dag $\mathrm{kg}^{-1}$; $\mathrm{P}$ de 1,4; $\mathrm{K}$ de 10 ( $\left.\mathrm{mg} \mathrm{dm}^{-3}\right)$; Ca de 0,5; $\mathrm{Mg}$ de 0,2; $\mathrm{Al}$ de

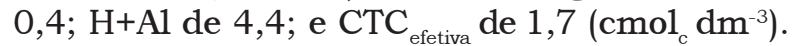
Para adequação do substrato quanto à nutrição, foram aplicados o equivalente a $150 \mathrm{mg} \mathrm{dm}^{-3} \mathrm{de}$ calcário dolomítico, $110 \mathrm{mg} \mathrm{dm}^{-3}$ de superfosfato simples e $20 \mathrm{mg} \mathrm{dm}^{-3}$ de cloreto de potássio. A adubação nitrogenada foi realizada em cobertura aos 30 dias após a emergência da cultura, na dose de $20 \mathrm{mg} \mathrm{dm}^{-3} \mathrm{de}$ ureia previamente dissolvida em água. As irrigações foram realizadas conforme a necessidade da cultura, por sistema de microaspersão.

O delineamento experimental utilizado foi em blocos ao acaso com quatro repetições, e as unidades experimentais foram constituídas por vaso com capacidade de $12 \mathrm{dm}^{3}$, preenchido com o substrato. Adotou-se arranjo fatorial em esquema $5 \times 5$, sendo cinco doses do mesotrione $\left(0,72,108,144\right.$ e $216 \mathrm{~g}$ ha $\left.^{1}\right)$ e cinco cultivares de mandioca: Coimbra, Platina, IAC 12, Coqueiro e Cacau-UFV.

O plantio das manivas nos vasos foi realizado no mês de março de 2011, com emergência visível cinco dias após o plantio (DAP). A aplicação do herbicida foi feita aos 30 DAP com pulverizador costal pressurizado a $\mathrm{CO}_{2}$, equipado com dois bicos TTI 110.02, espaçados de $0,5 \mathrm{~m}$ de largura, mantidos em pressão de $200 \mathrm{kPa}$ e volume de calda de $150 \mathrm{~L} \mathrm{ha}^{-1}$.

Aos 45 dias após a aplicação do herbicida, foram realizadas as avaliações fisiológicas, no terço superior da planta, na quarta folha completamente expandida. Utilizou-se o analisador de gases no infravermelho (IRGA), marca ADC, modelo LCA PRO+ (Analytical Development Co. Ltd, Hoddesdon, UK), em ambiente protegido, 
permitindo livre circulação do ar. As avaliações foram feitas entre 7 e $10 \mathrm{~h}$, de forma que fossem mantidas as condições ambientais homogêneas durante a avaliação de cada bloco. Foram avaliados o $\mathrm{CO}_{2}$ consumido $(\Delta C$ $\left.\mu \mathrm{mol} \mathrm{mol}{ }^{-1}\right)$, carbono interno $\left(C \mathrm{i}-\mu \mathrm{mol} \mathrm{mol}^{-1}\right)$, taxa fotossintética $\left(A-\mu \mathrm{mol} \mathrm{m} \mathrm{m}^{-2} \mathrm{~s}^{-1}\right)$, condutância estomática de vapores de água $\left(g \mathrm{~s}-\mathrm{mol} \mathrm{m}^{-1} \mathbf{s}^{-1}\right)$, taxa de transpiração $(E$ mol $\mathrm{H}_{2} \mathrm{O} \mathrm{m}^{-2} \mathrm{~s}^{-1}$ ) e temperatura da folha $\left(T F-{ }^{\circ} \mathrm{C}\right)$, bem como calculada a eficiência do uso da água (EUA - mol CO $\mathrm{Col} \mathrm{H}_{2} \mathrm{O}^{-1}$ ), obtida pela relação entre quantidade de $\mathrm{CO}_{2}$ fixado pela fotossintese e quantidade de água transpirada.

Os dados foram submetidos à análise de variância pelo teste $\mathrm{F}$; em caso de significância, as médias foram comparadas pelo teste de Tukey a $5 \%$ de probabilidade.

\section{RESULTADOS E DISCUSSÃO}

$\mathrm{O}$ consumo de $\mathrm{CO}_{2}(\Delta C)$ pela fotossintese no período de avaliação, para os cultivares de mandioca IAC-12, Coqueiro e Cacau-UFV, foi reduzido a partir da dose de $108 \mathrm{~g} \mathrm{ha}^{-1}$ do mesotrione (Tabela 1). Os genótipos IAC-12 e Coqueiro foram os que apresentaram as maiores reduções $(37,2$ e $41,9 \%$, respectivamente) no consumo de $\mathrm{CO}_{2}$ quando submetidos à maior dose do herbicida, em relação à testemunha.

As menores taxas de $\Delta C$, nos cultivares IAC-12 e Coqueiro, estão diretamente relacionadas com as maiores reduções na taxa fotossintética verificadas nesses cultivares. De modo geral, quanto mais acelerado for o metabolismo da planta, maior o $\Delta C$ por unidade de tempo (Galon et al., 2010), embora a capacidade de condução e trocas gasosas pelos estômatos seja considerada a principal limitação da assimilação de $\mathrm{CO}_{2}$ fotossintético (Hutmacher \& Krieg, 1983).

Semelhante ao observado para $\Delta C$ (Tabela 1$)$, a concentração de carbono interno $(C \mathrm{i})$ do cultivar Platina não foi afetada pela a aplicação do mesotrione (Tabela 2). O herbicida promoveu aumento na concentração de $C i$ nos genótipos Coimbra e IAC-12 a partir da dose de $144 \mathrm{~g} \mathrm{ha}^{-1}$ e para Coqueiro e Cacau-UFV na maior dose. As menores taxas de $\Delta C$ pela fotossintese nesses cultivares, quando tratados com mesotrione (Tabela 1), justificam o aumento na concentração de $C i$ no mesofilo foliar (Tabela 2). A Ci é considerada uma variável fisiológica influenciada por fatores ambientais, como disponibilidade hídrica, luz e energia, entre outros (Ometto et al., 2003). Como neste experimento o déficit hídrico e o sombreamento não foram fatores limitantes ao metabolismo das plantas de mandioca, acredita-se que os resultados sejam atribuídos ao efeito do herbicida no metabolismo de assimilação do carbono.

Em relação à taxa transpiratória $(E)$, houve efeito negativo na transpiração para IAC-12, Coimbra e Cacau-UFV quando receberam a aplicação do herbicida (Tabela 3). No entanto, o mesmo não foi constatado para os cultivares Platina e Coqueiro, que mantiveram valores semelhantes aos da testemunha.

A mandioca apresenta controle estomático eficiente sobre as trocas gasosas, a fim de reduzir as perdas de água quando as plantas são sujeitas a déficit hídrico ou a altas demandas evaporativas da atmosfera, protegendo assim as folhas de uma desidratação severa

Tabela $1-\mathrm{CO}_{2}$ consumido $\left(\triangle C-\mu \mathrm{mol} \mathrm{mol}{ }^{-1}\right)$ dos cultivares de mandioca, tratados com doses crescentes de mesotrione

\begin{tabular}{|l|c|c|c|c|c|}
\hline \multirow{2}{*}{\multicolumn{1}{c|}{ Cultivar }} & \multicolumn{5}{|c|}{ Mesotrione $\left(\mathrm{g} \mathrm{ha}^{-1}\right)$} \\
\cline { 2 - 6 } & 0 & 72 & 108 & 144 & 216 \\
\hline Coimbra & $43,40 \mathrm{aA}^{*}$ & $42,95 \mathrm{bA}$ & $38,30 \mathrm{bA}$ & $30,90 \mathrm{bB}$ & $30,10 \mathrm{bB}$ \\
\hline Platina & $56,35 \mathrm{aA}$ & $55,30 \mathrm{aA}$ & $58,45 \mathrm{aA}$ & $55,90 \mathrm{aA}$ & $59,35 \mathrm{aA}$ \\
\hline IAC-12 & $55,20 \mathrm{aA}$ & $52,90 \mathrm{aA}$ & $43,35 \mathrm{bB}$ & $41,55 \mathrm{abB}$ & $34,65 \mathrm{abC}$ \\
\hline Coqueiro & $56,95 \mathrm{aA}$ & $53,50 \mathrm{aA}$ & $44,15 \mathrm{bB}$ & $48,95 \mathrm{abB}$ & $33,05 \mathrm{bC}$ \\
\hline Cacau-UFV & $53,05 \mathrm{aA}$ & $53,40 \mathrm{aA}$ & $42,40 \mathrm{bB}$ & $40,60 \mathrm{abB}$ & $39,65 \mathrm{abB}$ \\
\hline CV $(\%)$ & & 27,81 & & \\
\hline
\end{tabular}

Médias seguidas pela mesma letra, minúscula na coluna e maiúscula na linha, não diferem entre si pelo teste de Tukey a 5\% de probabilidade. 
Tabela 2 - Carbono interno $\left(\mathrm{Ci}-\mu \mathrm{mol} \mathrm{mol}{ }^{-1}\right)$ dos cultivares de mandioca, tratados com doses crescentes de mesotrione

\begin{tabular}{|l|c|c|c|c|c|}
\hline \multirow{2}{*}{\multicolumn{1}{c|}{ Cultivar }} & \multicolumn{5}{|c|}{ Mesotrione $\left(\mathrm{g} \mathrm{ha}^{-1}\right)$} \\
\cline { 2 - 6 } & 0 & 72 & 108 & 144 & 216 \\
\hline Coimbra & $253,95 \mathrm{cB}^{*}$ & $266,45 \mathrm{aB}$ & $263,90 \mathrm{aB}$ & $275,20 \mathrm{abA}$ & $281,80 \mathrm{bA}$ \\
\hline Platina & $275,65 \mathrm{aA}$ & $261,95 \mathrm{aA}$ & $252,55 \mathrm{bA}$ & $261,35 \mathrm{bA}$ & $256,15 \mathrm{cA}$ \\
\hline IAC-12 & $266,70 \mathrm{abB}$ & $266,00 \mathrm{aB}$ & $272,50 \mathrm{aAB}$ & $280,65 \mathrm{aA}$ & $291,25 \mathrm{aA}$ \\
\hline Coqueiro & $253,30 \mathrm{cB}$ & $257,60 \mathrm{aB}$ & $259,05 \mathrm{abB}$ & $258,05 \mathrm{bB}$ & $296,25 \mathrm{aA}$ \\
\hline Cacau-UFV & $265,40 \mathrm{abB}$ & $265,00 \mathrm{aB}$ & $268,45 \mathrm{aB}$ & $258,65 \mathrm{aB}$ & $290,35 \mathrm{aA}$ \\
\hline CV (\%) & & & 10,64 & & \\
\hline
\end{tabular}

Médias seguidas pela mesma letra, minúscula na coluna e maiúscula na linha, não diferem entre si pelo teste de Tukey a 5\% de probabilidade.

Tabela 3 - Taxa transpiratória $\left(E-\mu \mathrm{mol} \mathrm{H}_{2} \mathrm{O} \mathrm{m}^{-2} \mathrm{~s}^{-1}\right)$ dos cultivares de mandioca, tratados com doses crescentes de mesotrione

\begin{tabular}{|l|c|c|c|c|c|}
\hline \multirow{2}{*}{ Cultivar } & \multicolumn{5}{|c|}{ Mesotrione $\left(\mathrm{g} \mathrm{ha}^{-1}\right)$} \\
\cline { 2 - 6 } & 0 & 72 & 108 & 144 & 216 \\
\hline Coimbra & $3,52 \mathrm{abA}^{*}$ & $3,43 \mathrm{aA}$ & $2,67 \mathrm{bB}$ & $2,73 \mathrm{bB}$ & $2,52 \mathrm{bB}$ \\
\hline Platina & $4,16 \mathrm{aA}$ & $3,75 \mathrm{aAB}$ & $3,37 \mathrm{aA}$ & $3,54 \mathrm{aA}$ & $3,47 \mathrm{aA}$ \\
\hline IAC-12 & $3,73 \mathrm{abA}$ & $3,71 \mathrm{aA}$ & $3,08 \mathrm{abAB}$ & $3,22 \mathrm{aAB}$ & $2,77 \mathrm{abB}$ \\
\hline Coqueiro & $3,17 \mathrm{bA}$ & $3,43 \mathrm{aA}$ & $3,27 \mathrm{aA}$ & $3,41 \mathrm{abA}$ & $3,03 \mathrm{aA}$ \\
\hline Cacau-UFV & $4,17 \mathrm{aA}$ & $3,88 \mathrm{aA}$ & $3,68 \mathrm{aA}$ & $3,65 \mathrm{aA}$ & $3,20 \mathrm{aB}$ \\
\hline CV $(\%)$ & & & 20,84 & & \\
\hline
\end{tabular}

Médias seguidas pela mesma letra, minúscula na coluna e maiúscula na linha, não diferem entre si pelo teste de Tukey a 5\% de probabilidade.

(E1-Sharkawy, 2007). Dessa forma, pode-se inferir que as menores taxas de transpiração verificadas nesses genótipos relacionam-se ao efeito do mesotrione na condutância estomática.

A condutância estomática ( $g$ s) está relacionada com a abertura dos estômatos, ou seja, a resistência associada à difusão de $\mathrm{CO}_{2}$ pela abertura estomática. Para todos os cultivares, exceto o Platina, a gs diminuiu a partir da dose de $108 \mathrm{~g} \mathrm{ha}^{-1}$ (Tabela 4). A menor gs promoveu maior resistência à difusão dos gases; com isso, uma menor taxa de transpiração provocada pela redução da abertura estomática pode ser observada na Tabela 3. As mudanças na resistência estomática são importantes para a regulação da perda de água pela planta e para o controle da taxa de absorção de dióxido de carbono necessário à fixação contínua durante a fotossintese (Taiz \& Zeiger, 2009). Independentemente da dose, o cultivar Coimbra apresentou os menores valores de gs em relação aos demais, demonstrando maior resistência à difusão dos gases e, consequentemente, menor transpiração e fotossíntese, respectivamente (Tabelas 3 e 5).
Quanto maior a taxa fotossintética da espécie, mais rapidamente o $\mathrm{CO}_{2}$ é consumido, e menor sua concentração no interior da folha, supondo-se que os estômatos estejam fechados (Corniani et al., 2006). O consumo de $\mathrm{CO}_{2}$ amplia as diferenças na concentração desse gás entre a parte interna da folha e o ambiente externo; via de regra, quanto maior for este gradiente $(\Delta C)$, mais rapidamente o $\mathrm{CO}_{2}$ entrará na folha após a abertura estomática, em função do maior gradiente de concentração.

O mesotrione causou redução das taxas fotossintéticas de todos os cultivares de mandioca nas doses de 144 e $216 \mathrm{~g} \mathrm{ha}^{-1}$, quando comparado com o tratamento sem aplicação (Tabela 5). No entanto, Cacau-UFV e IAC-12 apresentaram redução na fotossintese a partir da dose de $108 \mathrm{~g} \mathrm{ha}^{-1}$. As maiores taxas de reduções fotossintéticas foram verificadas nos cultivares Coqueiro e IAC-12, respectivamente. Ao observar a concentração de carbono interno $(C \mathrm{i})$, verifica-se que foram eles que apresentaram maior acúmulo de $\mathrm{CO}_{2}$ interno no mesofilo (Tabela 2). Isso demonstra que o herbicida mesotrione alterou o metabolismo de assimilação de carbono, reduzindo a taxa 
fotossintética das plantas de mandioca, além de reduzir também a condutância estomática, fazendo com que houvesse diminuição do grau de abertura estomática (Tabela 4), aumentando o incremento de $\mathrm{CO}_{2}$ interno. Em condições ambientais em que ocorra uma situação de estresse, as plantas de mandioca reduzem suas taxas fotossintéticas como forma de prevenir maiores danos (El-Sharkawy, 2003). Isso, provavelmente, reduz a condutância estomática, que diminui o influxo de $\mathrm{CO}_{2}$ no mesofilo foliar.
Os resultados da eficiência do uso da água (EUA) demonstram que somente Coqueiro teve redução nessa variáve1, ocasionada pela aplicação do herbicida (Tabela 6). Essa redução pode ser em função da redução na fotossintese da planta, causando em última instância redução no acúmulo de biomassa devido à intoxicação pelo herbicida. A EUA é caracterizada como a quantidade de água transpirada por uma cultura para a produção de certa quantidade de matéria seca (Silva et al., 2007). Por isso, é altamente influenciada pelo tipo de

Tabela 4 - Condutância estomática $\left(g \mathrm{~s}-\mu \mathrm{mol} \mathrm{m} \mathrm{m}^{-1} \mathrm{~s}^{-1}\right)$ dos cultivares de mandioca, tratados com doses crescentes de mesotrione

\begin{tabular}{|c|c|c|c|c|c|}
\hline \multirow{2}{*}{ Cultivar } & \multicolumn{5}{|c|}{ Mesotrione $\left(\mathrm{g} \mathrm{ha}^{-1}\right)$} \\
\hline & 0 & 72 & 108 & 144 & 216 \\
\hline Coimbra & $0,33 \mathrm{bA}^{*}$ & $0,36 \mathrm{bA}$ & $0,24 \mathrm{bB}$ & $0,23 \mathrm{bB}$ & $0,25 \mathrm{bB}$ \\
\hline Platina & $0,55 \mathrm{aA}$ & $0,54 \mathrm{aA}$ & $0,47 \mathrm{aA}$ & $0,54 \mathrm{aA}$ & $0,67 \mathrm{aA}$ \\
\hline IAC-12 & $0,56 \mathrm{aA}$ & $0,58 \mathrm{aA}$ & $0,49 \mathrm{aAB}$ & $0,47 \mathrm{abAB}$ & $0,39 \mathrm{abB}$ \\
\hline Coqueiro & $0,42 \mathrm{abA}$ & $0,42 \mathrm{abA}$ & $0,43 \mathrm{aA}$ & $0,40 \mathrm{abA}$ & $0,27 \mathrm{bB}$ \\
\hline Cacau-UFV & $0,53 \mathrm{aA}$ & $0,52 \mathrm{aA}$ & $0,50 \mathrm{aA}$ & $0,47 \mathrm{abA}$ & $0,43 \mathrm{abB}$ \\
\hline $\mathrm{CV}(\%)$ & \multicolumn{5}{|c|}{32,13} \\
\hline
\end{tabular}

Médias seguidas pela mesma letra, minúscula na coluna e maiúscula na linha, não diferem entre si pelo teste de Tukey a 5\% de probabilidade.

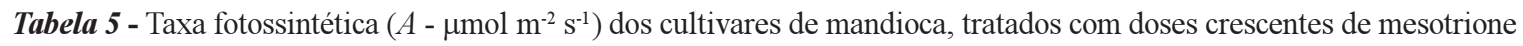

\begin{tabular}{|l|c|c|c|c|c|}
\hline \multirow{2}{*}{\multicolumn{1}{c|}{ Cultivar }} & \multicolumn{5}{|c|}{ Mesotrione $\left(\mathrm{g} \mathrm{ha}^{-1}\right)$} \\
\cline { 2 - 6 } & 0 & 72 & 108 & 144 & 216 \\
\hline Coimbra & $12,45 \mathrm{bA}^{*}$ & $15,28 \mathrm{bA}$ & $12,22 \mathrm{bA}$ & $9,87 \mathrm{bB}$ & $9,74 \mathrm{bB}$ \\
\hline Platina & $17,13 \mathrm{aA}$ & $17,68 \mathrm{aA}$ & $16,28 \mathrm{aA}$ & $14,17 \mathrm{aB}$ & $14,00 \mathrm{aB}$ \\
\hline IAC-12 & $17,19 \mathrm{aA}$ & $17,64 \mathrm{aA}$ & $11,73 \mathrm{bB}$ & $12,11 \mathrm{abB}$ & $10,78 \mathrm{abB}$ \\
\hline Coqueiro & $17,24 \mathrm{aA}$ & $17,05 \mathrm{aA}$ & $17,04 \mathrm{aA}$ & $14,37 \mathrm{aAB}$ & $10,54 \mathrm{abB}$ \\
\hline Cacau-UFV & $16,21 \mathrm{aA}$ & $15,52 \mathrm{bA}$ & $13,28 \mathrm{bB}$ & $13,96 \mathrm{abB}$ & $12,61 \mathrm{abB}$ \\
\hline CV $(\%)$ & & & 21,27 & & \\
\hline
\end{tabular}

Médias seguidas pela mesma letra, minúscula na coluna e maiúscula na linha, não diferem entre si pelo teste de Tukey a 5\% de probabilidade.

Tabela 6 - Eficiência do uso da água $\left(E U A\right.$ - $\left.\mu \mathrm{mol} \mathrm{CO}_{2} \mathrm{~mol} \mathrm{H}_{2} \mathrm{O}^{-1}\right)$ dos cultivares de mandioca, tratados com doses crescentes de mesotrione

\begin{tabular}{|l|c|c|c|c|c|}
\hline \multirow{2}{*}{ Cultivar } & \multicolumn{5}{|c|}{ Mesotrione $\left(\mathrm{g} \mathrm{ha}^{-1}\right)$} \\
\cline { 2 - 6 } & 0 & 72 & 108 & 144 & 216 \\
\hline Coimbra & $3,51 \mathrm{bA}^{*}$ & $4,47 \mathrm{aA}$ & $4,55 \mathrm{aA}$ & $3,67 \mathrm{bA}$ & $3,94 \mathrm{abA}$ \\
\hline Platina & $4,29 \mathrm{abA}$ & $4,71 \mathrm{aA}$ & $4,86 \mathrm{aA}$ & $4,19 \mathrm{aA}$ & $4,06 \mathrm{aA}$ \\
\hline IAC-12 & $4,64 \mathrm{abA}$ & $4,81 \mathrm{aA}$ & $4,07 \mathrm{abA}$ & $4,06 \mathrm{abA}$ & $4,08 \mathrm{aA}$ \\
\hline Coqueiro & $5,72 \mathrm{aA}$ & $5,03 \mathrm{aA}$ & $5,23 \mathrm{aA}$ & $4,37 \mathrm{aAB}$ & $3,41 \mathrm{bB}$ \\
\hline Cacau-UFV & $4,09 \mathrm{aA}$ & $4,00 \mathrm{aA}$ & $3,74 \mathrm{bA}$ & $3,90 \mathrm{bA}$ & $3,60 \mathrm{bA}$ \\
\hline CV $(\%)$ & & 25,22 & & \\
\hline
\end{tabular}

Médias seguidas pela mesma letra, minúscula na coluna e maiúscula na linha, não diferem entre si pelo teste de Tukey a 5\% de probabilidade. 
Tabela 7 - Temperatura da folha $\left(T F-{ }^{\circ} \mathrm{C}\right)$ dos cultivares de mandioca, tratados com doses crescentes de mesotrione

\begin{tabular}{|l|c|c|c|c|c|}
\hline \multirow{2}{*}{ Cultivar } & \multicolumn{5}{|c|}{ Mesotrione $\left(\mathrm{g} \mathrm{ha}^{-1}\right)$} \\
\cline { 2 - 6 } & 0 & 72 & 108 & 144 & 216 \\
\hline Coimbra & $30,23 \mathrm{aA}^{*}$ & $29,48 \mathrm{aA}$ & $29,55 \mathrm{aA}$ & $29,59 \mathrm{aA}$ & $29,50 \mathrm{aA}$ \\
\hline Platina & $30,26 \mathrm{aA}$ & $29,66 \mathrm{aA}$ & $29,77 \mathrm{aA}$ & $29,28 \mathrm{aA}$ & $29,15 \mathrm{aA}$ \\
\hline IAC-12 & $29,45 \mathrm{aA}$ & $29,92 \mathrm{aA}$ & $30,98 \mathrm{aA}$ & $29,73 \mathrm{aA}$ & $29,97 \mathrm{aA}$ \\
\hline Coqueiro & $29,39 \mathrm{aA}$ & $29,85 \mathrm{aA}$ & $30,11 \mathrm{aA}$ & $29,64 \mathrm{aA}$ & $29,60 \mathrm{aA}$ \\
\hline Cacau-UFV & $29,21 \mathrm{aA}$ & $29,98 \mathrm{aA}$ & $29,16 \mathrm{aA}$ & $29,92 \mathrm{aA}$ & $29,34 \mathrm{aA}$ \\
\hline CV $(\%)$ & & 3,82 & & \\
\hline
\end{tabular}

Médias seguidas pela mesma letra, minúscula na coluna e maiúscula na linha, não diferem entre si pelo teste de Tukey a 5\% de probabilidade.

metabolismo do carbono da espécie e por outras características morfofisiológicas, como densidade e abertura dos estômatos, revestimento da superfície foliar (presença de tricomas, cerosidade nas folhas) e posicionamento dos estômatos em relação à superfície da folha (planos ou em cavidades). Aspiazú et al. (2010) trabalharam com a mandioca em condição de competição e verificaram que a cultura apresentou maior EUA comparada à das plantas daninhas testadas. A EUA é também influenciada pelos fatores ambientais, como temperatura da folha, velocidade do vento e umidade relativa do ar. Quando sob condições de temperatura moderada, as plantas de mandioca normalmente são muito eficientes quanto ao uso da água, ou seja, perdem menos água que muitas espécies, para a fixação da mesma quantidade de $\mathrm{CO}_{2}$ (E1 - Sharkawy, 2003).

Uma vez que a temperatura da folha $(T F)$ não mostrou significância estatística para os cultivares (Tabela 7), pode-se supor que o metabolismo não foi seriamente afetado e que esse fator provavelmente possa ser excluído da participação na gs. O metabolismo do vegetal incrementa a $T F$ a tal ponto que, via de regra, é superior à temperatura do ar ao seu redor, que é dependente da taxa de transpiração. Assim, o aumento do metabolismo pode ser indiretamente aferido em função do gradiente entre a $T F$ e a temperatura do ar. Normalmente essa diferença é de somente um ou dois graus, porém, em casos extremos, pode exceder $5^{\circ} \mathrm{C}$ (Atkin et al., 2000; Taiz \& Zeiger, 2009).

A aplicação do herbicida causou reduções nas taxas fotossintéticas de todas as plantas e no uso eficiente da água apenas para o
Coqueiro. No entanto, a temperatura da folha não foi alterada em nenhum cultivar. Assim, foi possivel inferir que os cultivares avaliados apresentaram diferentes niveis de tolerância ao mesotrione.

\section{AGRADECIMENTOS}

Ao Conselho Nacional de Desenvolvimento Científico e Tecnológico - CNPq e à Fundação de Amparo à Pesquisa do Estado de Minas Gerais - FAPEMIG, pelo auxílio financeiro ao projeto; e à Coordenação de Aperfeiçoamento de Pessoal de Nível Superior (Capes), pela concessão de bolsa ao primeiro autor.

\section{LITERATURA CITADA}

\section{ASSOCIAÇÃO BRASILEIRA DOS PRODUTORES DE AMIDO DE MANDIOCA - ABAM. Registro de defensivos agrícolas para a mandioca começa a se concretizar. Disponível em: <http://www.abam.com.br/ includes $/$ menu3/noticias_imp.php?id=353>. Acesso em: 10 dez. 2011}

ASPIAZÚ, I. et al. Eficiência do uso da água de plantas de mandioca em condições de competição. Planta Daninha, v. 28 , n. 4 , p. $699-703,2010$

ATKIN, O. K. et al. Leaf respiration of snow gum in the light and dark. Interactions between Temperature and Irradiance. Plant Physiol., v. 122, n. 3, p. 915-923, 2000 .

BAPTISTA, J. M. et al. Programa nacional para o uso eficiente da água. Lisboa: Instituto Superior de Agronomia, 2001. 212 p.

BIFFE, D. F. et al. Avaliação de herbicidas para dois cultivares de mandioca. Planta Daninha, v. 28, n. 4, p. $807-816,2010$. 
BRASIL. Ministério da Agricultura, Pecuária e

Abastecimento. Disponível em: $<$ http://

www.agricultura.gov.br>. Acesso em: 10 jan. 2012.

CORNIANI, N. et al. Determinação das trocas gasosas e de potencial hídrico através do uso de sistemas portáteis na avaliação do estresse. In: SIMPÓSIO INTERNACIONAL DE INICIAÇÃO CIENTÍFICA DA UNIVERSIDADE DE SÃO PAULO, 14., 2006, Piracicaba. Anais... São Paulo: USP, 2006. CD-ROM

EL-SHARKAWY, M. A. Cassava biology and physiology.

Plant Molec. Biol., v. 53, n. 5, p. 481-501, 2003

EL-SHARKAWY, M. A. Physiological characteristics of cassava tolerance to prolonged drought in the tropics: Implications for breeding cultivars adapted to seasonally dry and semi arid environments. Braz. J. Plant Physiol., v. 19, n. 4, p. 257-286, 2007.

FOLONI, L. L. Callisto $®$ (mesotrione) - um novo herbicida pós-emergente para a cultura do milho (Zea mays L.). In CONGRESSO BRASILEIRO DA CIÊNCIA DAS PLANTAS DANINHAS, 23., 2002, Gramado. Resumos... Gramado: Sociedade Brasileira da Ciência das Plantas Daninhas, 2002. p. 308.

GALON, L. et al. Tolerância de novos genótipos de cana-deaçúcar a herbicidas. Planta Daninha, v. 28, n. 2, p. 329-338, 2010.

HUTMACHER, R. B.; KRIEG, D. R. Photosynthetic rate control in cotton. Plant Physiol., v. 73, n. 3, p. 658-661, 1983.
JOHANNS, O; CONTIERO, R. Efeitos de diferentes períodos de controle e convivência de plantas daninhas com a cultura da mandioca. R. Ci. Agron., v. 37, n. 3, p. 326-331, 2006.

LEE, D. L. The discovery and structural requirements of inhibitors of p-hydroyphenylpyruvate dioxygenase. Weed Sci., v. 45, n. 4, p. 601-609, 1997.

OMETTO, J. P. H. B. et al. Variação temporal do isótopo estável do carbono em material arbóreo em florestas da região Amazônica. In: CONGRESSO BRASILEIRO DE ECOLOGIA, 4., 2003, Fortaleza. Anais... Rio Claro: Sociedade de Ecologia do Brasil, 2003.

PROCÓPIO, S. O. et al. Ponto de murcha permanente de soja, feijão e plantas daninhas. Planta Daninha, v. 22, n. 1, p. $35-41,2004$

SILVA, A. A. et al. Biologia de plantas daninhas. In : Tópicos em manejo de plantas daninhas. Viçosa, MG: Universidade Federal de Viçosa, cap. 3, 2007. p. 58-117.

SILVEIRA, H. M. et al. Sensibilidade de cultivares de mandioca ao mesotrione. R. Bras. Herbic., v.11, n. 1, p. 24-31, 2012.

SYNGENTA FOUNDATION. Disponível em: $<\mathrm{http}: / /$ www.syngenta.com.br>. Acesso em: 10 jan. 2012

TAIZ, L.; ZEIGER, E. Fisiologia vegetal. 4.ed. Porto Alegre: Artmed, 2009. 848 p

WICHERT, R. A. et al. Technical overview of ZA1296, a new corn herbicide from ZENECA. Weed Sci. Soc. Am. Abstr., n. 39, p. 65,1999 